\title{
Predicting Sky Dome Appearance on Earth-like Extrasolar Worlds
}

\author{
Alexander Wilkie* \\ Charles University in Prague
}

\author{
Lukas Hošek ${ }^{\dagger}$ \\ Charles University in Prague
}

\begin{abstract}
In this paper, we present a case study on how, given some reasonable assumptions, a sky dome model originally developed for terrestrial Predictive Rendering purposes can be re-used to give a scientifically plausible first impression of what outdoor scenes on earth-like exoplanets with a similar atmospheric structure would look like. On this very specific type of exoplanet, humans could reasonably be expected to survive without a space suit, and life-forms very similar to those found on Earth could theoretically evolve. This makes a simulation of typical outdoor lighting on this kind of planet interesting for various scientific (speculative exo-biological simulations) and not so scientific ("realistic" science fiction movie settings) reasons.

If the properties of the exoplanet itself are restricted to resemble Earth, the remaining free parameter are the sun(s) which the exoplanet orbits. In the course of our case study, we found that even for fairly different star types, the overall appearance of the sky does not change as much as one might assume at first. Also, even the skies of Earth-like worlds that orbit binary stars would have a fairly normal appearance, although specific oddities can be observed.
\end{abstract}

CR Categories: I.3.7 [Computer Graphics]: Three-Dimensional Graphics and Realism

Keywords: skylight models, predictive rendering

\section{Introduction}

Attempting to imagine the inhabitants and appearance of alien lands that no man had yet set foot in is an effort probably as old as our species. It certainly dates as far back as antiquity, with Pliny the Elder writing, apparently not from first hand knowledge, about the strange inhabitants of faraway countries: both headless and dog-headed men were featured in his great natural history of the world [Dennis 1995]. With varying frequency, such works, and references to strange creatures and places to be found in far away countries, continued to appear over the course of history - although perhaps not quite as often as it is sometimes imagined (cf. chapter 10 of [Moreland and Bannister 1989]).

In the 20th century, with our own planet being increasingly well and exhaustively cartographed, and with the advent of space flight and corresponding huge advances in astronomy, the attention of this particular branch of futuristic and speculative art firmly shifted towards entire new worlds, instead of unknown lands far away on our home planet: the field of Science Fiction was born. The quality of

*e-mail: wilkie@cgg.mff.cuni.cz

†e-mail: hosek@cgg.mff.cuni.cz works in this category is highly diverse, and falls in a huge range between whimsical notions of a largely cartoonish outer space populated with more or less repulsive aliens [Ashley 2000] (cf. the illustrations in figure 1) and serious futurism on par with the best of world literature, such as - to name but two classics - the works of Stanisław Lem, or Arthur C. Clarke. Also, with the advent of high quality cinematographic effects, more or less plausible and elaborate alien world scenarios, such as the one shown in figure 2 became increasingly popular as backdrops for futuristic movies.
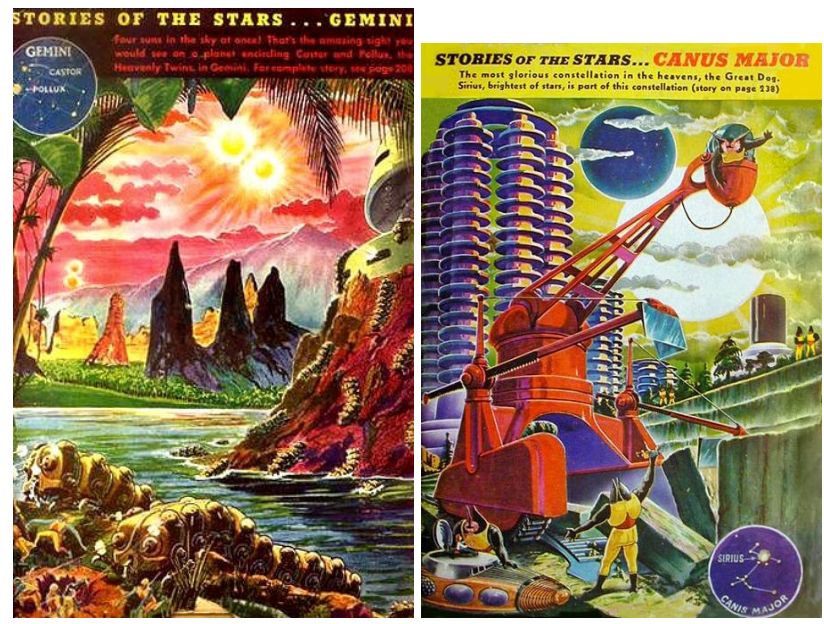

Figure 1: Exoplanet skies from mid-20th century science fiction pulp magazine covers Ashley 2000]. The lurid and improbable settings and colours in such images were usually chosen purely for effect, and in most cases little effort - if any - was invested in making the content of such images in any way scientifically plausible. Images from the "Tales of Future Past" section of http://davidszondy.com/

In this paper, we focus on one particular sub-problem of "imagining alien worlds" - namely, the lighting conditions found on such planets. In particular, we present a physically plausible case study about the likely appearance of outdoor scenes of earth-like planets that orbit different stars. While it of course also falls, at least to some degree, into the realm of educated guesswork, our case study differs from purely speculative efforts in this direction insofar as our findings do have a physical foundation to stand on. As such, the results we obtained can not only serve to make computer graphics renderings of science fiction settings more physically plausible and realistic (which is a debatable effort in and by itself), but also might prove to be useful for actual scientific study in other fields, such as exobiology.

\section{Related Research}

Three points are of interest for our study: first, other studies of exoplanet appearance, second, the basics of star colour and emission, and third, analytical models of sky dome appearance. 

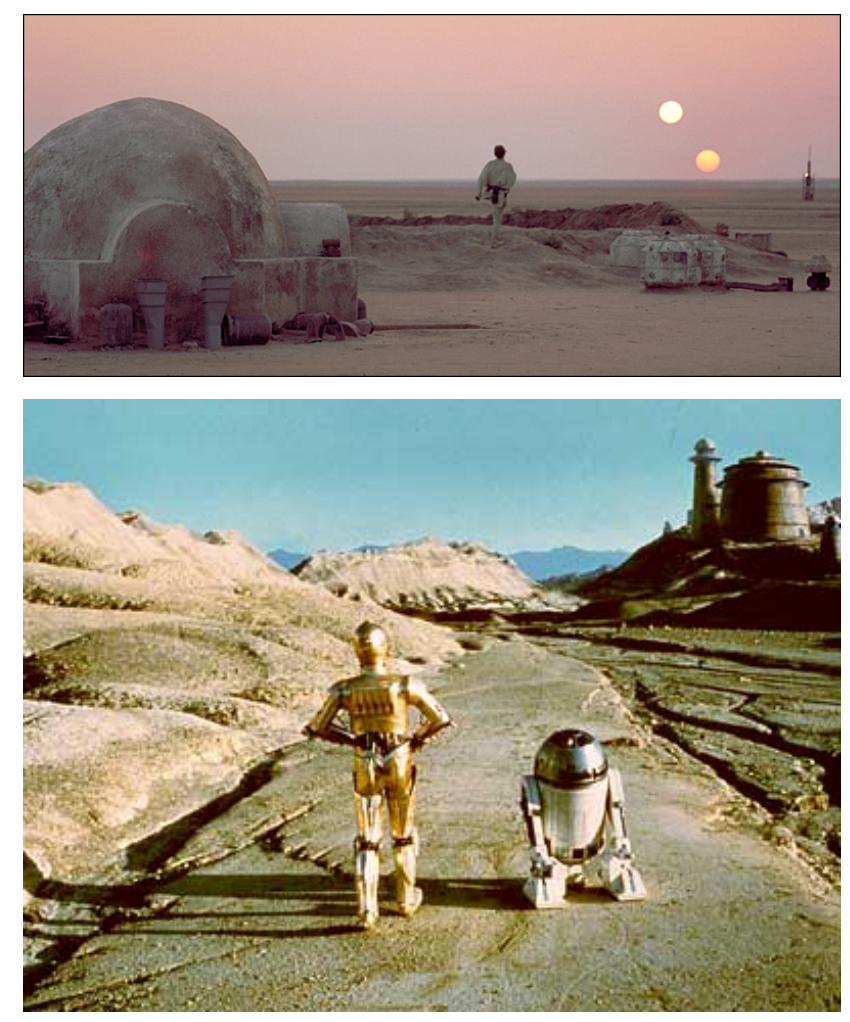

Figure 2: Exoplanet images from the 1977 movie Star Wars. Part of the movie is set on a rather earth-like, if somewhat more arid, exoplanet named Tatooine which orbits a close binary of differing colour: in supporting texts, these are identified as class $\mathrm{G}$ and $\mathrm{K}$ stars. The sunset scene shown above, into which the second sun was at the time inserted with analog cinematographic tricks, is an iconic image for the first movie in the series. But since outdoor photography for the movie was of course shot in terrestrial settings, scenes like the one shown beneath lack a feature that would be noticeable even for a close binary like the one shown here: the doubled shadow fringes which can be seen in our result rendering in figure 9 The blue sky colour, however, is actually fairly plausible, and would look much the same in "reality". (Images (C) Disney)

\subsection{Studies of Exoplanet Appearance}

Due to the scarcity of hard evidence about such locations, very little actual scientific work seems to have been conducted on the appearance of exoplanet sceneries. There are of course entire scientific institutions devoted to the study of exoplanets [NASA 2013], but as of today, the focus of these institutions still lies firmly in other areas, such as general planetary science.

However, the question of what the skies of an alien world might look like has intrigued at least one author to offer some informed suggestions [Freitas 1979|. While the cited book is on the whole not much concerned with object appearance and topics relevant to Computer Graphics, and while it does have - if only because of the basic topic it covers - a certain fringe science quality to it, section 5.4.2 does give a brief but technically sound and useful discussion of potential exoplanet sky appearance.

\subsection{Star Emission Spectra}

The visible cosmos is populated with a huge number of individual stars, practically all of which seem to adhere to certain basic rules as far as their overall emission and appearance is concerned. As a rough first approximation, the light emission of stars can be described by so-called blackbody radiation [Siegel and Howell 2001], in dependence of the surface temperature of the star. Per unit solid angle the radiation of such an object is, in dependence of wavelength $\lambda$ and temperature $T$, given by:

$$
I(\lambda, T)=\frac{2 h c^{2}}{\lambda^{5}} \frac{1}{e^{\frac{h c}{\lambda k T}}-1}
$$

Figure 3 shows the currently assumed distribution of star colours and luminosities found in the observable cosmos.

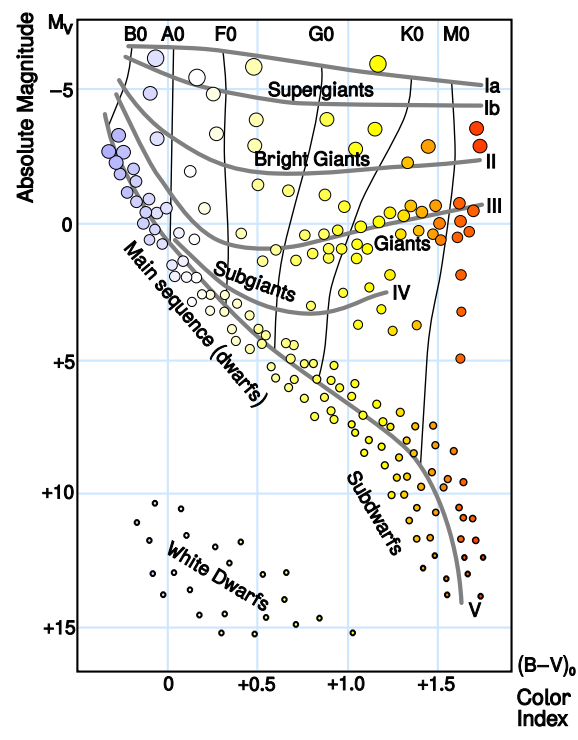

Figure 3: The Hertzsprung-Russell diagram, in which star luminosity and colour are correlated with relative abundance in the visible cosmos. It nicely illustrates that for physically plausible exoplanets, the choice of solar illumination is restricted to a comparatively narrow range of colours that ranges from blue-white to reddish-orange. (Image under Creative Commons license, Wikipedia)

Of course, the emission spectra of real stars are nowhere as simple as plain blackbody emission spectra, and a very complex research matter in their own right. In particular, star emission spectra can, on a small-scale spectral level, differ considerably even between stars which exhibit fairly similar colour for a human observer. However, while the differences between such spectra are highly significant from a scientific viewpoint (absorption lines yield crucial insights into the composition of stars), such emission spectra differences are mostly to be observed at the level of individual absorption lines. As a consequence, they are largely irrelevant for the prediction of the appearance of objects illuminated by such a sun to a human observer, and as such, can be ignored for the purposes of our study.

\subsection{Analytical Models of Sky-Dome Radiance}

In a renderer, three basic options exist to include atmospheric effects, and realistic sky dome appearance: 


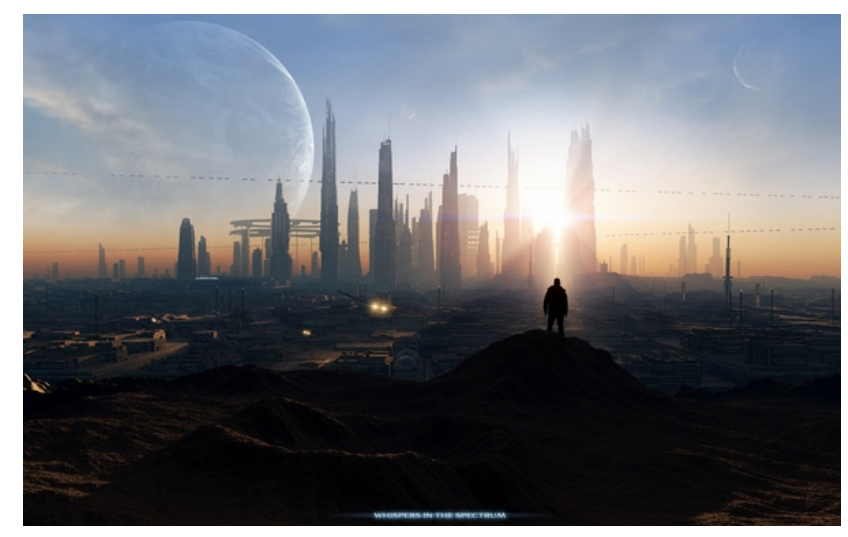

Figure 4: Contemporary computer-generated science fiction imagery of a cityscape on an alien planet. An earth-like solar radiance model is being used to great effect here. However, without accurate sky dome models for altered solar emission spectra, a similar shot for a significantly different parent star (say, for a much larger reddish sun) would involve artistic speculation, and might not look as convincing as far as subtle effects are concerned. (Image (c) http: //darink.deviantart.com)

1. Explicit simulation of light transport in the atmosphere.

2. High dynamic range (HDR) captures of real outdoor environments, used as backdrops for outdoor scenes.

3. Analytic models of sky dome luminance [Preetham et al. 1999, ISO/CIE 2004, Haber et al. 2005, Hosek and Wilkie 2012].

The first option, explicit simulation of light transport in the entire atmosphere, is not practical due to excessive computational requirements, and will in all likelihood remain impracticable for the foreseeable future. Which is most unfortunate, since it would offer possibilities for exoplanet simulations that go beyond what the option three that was used by us offers. The second option, HDR captures of real outdoor environments, is of course very practical for normal graphics uses - but obviously not suitable for prediction of exoplanet environments.

It has to be stressed that option three, analytical sky dome models, are also not generally useful for the prediction of arbitrary exoplanet environments. Typically, such models involve a large amount of pre-computation of light scattering effects in the atmosphere to avoid the problems of option one: excessive computational cost at run time. Since these pre-computations are specific for the terrestrial atmosphere (and usually, also the terrestrial sun), this renders such models more or less useless for our intended purpose.

There is one potential exception, though: both the Preetham [Preetham et al. 1999] and the Hošek [Hosek and Wilkie 2012] models compute sky dome luminance patterns and solar radiance independently for each waveband, and can therefore be used to predict the appearance of the sky on an Earth-like planet (but only that!) under a different sun. For our experiments, we opted to use the Hošek model, as it also includes the near UV range, which can significantly affect the appearance of materials that include optical brighteners, and that also plays a significant role in biological mechanisms.

\section{Adapting the Hošek Sky Model for Exo- planet Scenes}

When using the Hošek model to predict the appearance of an Earthlike exoplanet outdoor scene, slightly different considerations apply for the adaptation of the sky dome luminance patterns, and the direct solar radiance model.

Common to both is the already stated observation that due to its spectral nature, the Hošek model predicts sky dome luminance patterns and solar radiance independently for each waveband. Since the intensity of each waveband is solely dependent on the input radiance from the star that the world in question is orbiting, it is trivial to re-scale the wavebands to match a different star radiance. For a given star temperature $T$, each wave-band $i(i \in[0 . .11]$, as per the definition of the Hošek model), the ratio between the spectral blackbody emission according to equation 1 for this wavelength, and the original solar radiance $S_{i}$ for this waveband used in the brute force simulations (i.e. the original input intensity for this waveband) is computed:

$$
f_{i}=\frac{I\left(\lambda_{i}, T\right)}{S_{i}}
$$

Values for $S_{i}$ are given in [Preetham et al. 1999] and also in the source code distributed with [Hosek and Wilkie 2013]. This factor $f_{i}$ is then used to scale the intensity of all wavebands $i$ that occur in the model, both for solar radiance and sky dome luminance. However, further consideration has also to be given to the size of the solar disc on the sky dome, and the relative brightness scale of sky dome and solar disc.

\subsection{Direct Solar Radiance}

When adapting the model for direct solar radiance, one has to bear in mind that there is very likely a comparatively tight range of absolute solar irradiance values for which an earth-like planet with an atmosphere like the one we assume in our model can exist in the first place. Too much irradiance, and the atmosphere likely boils off into space, too little, it freezes and disappears as well. Which means that stars of considerably different emission colour and intensity than our sun will have to be fairly different in size from it (in terms of how large they appear on the sky dome overhead), to still provide a reasonable and inhabitable amount of irradiance. Red stars will need to be much larger on the sky dome than our sun, while white or blue stars will have to be comparatively tiny.

In order to cover this effect, we have to compute a plausible solar diameter for a given emission spectrum. We do this by first computing $f_{\text {avg }}$, the average of the correction factors $f_{i}$, for the visible part of the spectrum (and only that - the two UV bands are intentionally omitted). We then scale the diameter of the solar disc on the exoplanet sky $r_{E}$ according to the formula

$$
r_{E}=\frac{r_{T}}{\sqrt{f_{\text {avg }}}}
$$

with $r_{T}$ being the solar diameter on the terrestrial sky: $0.51^{\circ}$. This shrinks or expands the solar disc so that the average energy over the visible range is roughly the same as for the terrestrial sun.

In addition to the re-sizing of the solar disc, each waveband $i$ of the solar radiance function provided by the second part of the Hošek model [Hosek and Wilkie 2013] has of course to be scaled by the appropriate factor $f_{i}$.

With respect to the re-sizing of the solar disc, it should be kept in mind that the sky dome luminance patterns provided by the Hošek 
model are not parameterised by solar diameter - i.e. the sky dome patterns stay exactly the same for different solar diameters. Which is of course not correct. But from experimental experience with brute force calculations of sky dome luminance patterns we know that solar discs up to several degrees in diameter $(!$ - our own sun is half a degree across) do not cause the luminance patterns on the sky to change perceptibly. The reason we noticed this is that we initially used unrealistically large suns in our own brute force sky dome path tracer, in order to improve convergence speeds. Later, we managed to do the reference renderings much faster even with realistically small suns, and found that there was no real difference in sky dome appearance anyway. Which has led us to conclude that for the purposes of an approximative case study like this, re-use of terrestrial luminance patterns is permissible as long as the solar disc stays within reasonable bounds - say, $<\approx 5^{\circ}$.

\subsection{Sky Dome Luminance Patterns}

Since we re-scaled the solar disc so that the alien sun has an absolute brightness that is reasonably close to that of the terrestrial sun, we also have to scale the sky dome luminance values by the uniform factor $f_{\text {avg }}$ across all wavebands to match this. So for each waveband $i$, the resulting exoplanet sky dome luminance $\mathbb{F}_{E}$ becomes

$$
\mathbb{F}_{E i}(\theta, \gamma)=\frac{\mathbb{F}(\theta, \gamma)_{i} \cdot f_{i}}{f_{\text {avg }}},
$$

with $\mathbb{F}(\theta, \gamma)_{i}$ being the output of the original Hošek model for that waveband.

\section{Results}

For our case study, we assumed the emission of all stars that illuminate an Earth-like exoplanet to be a perfect blackbody emission spectrum (as outlined in section 2.2. This is never entirely realistic - real star emission spectra are considerably more complex than this, mainly due to absorption effects in the outer layers of stars. However, blackbody spectra are a reasonable first assumption in a usage scenario like this, where $100 \%$ accuracy is simply not necessary: for rendering purposes, there are likely no visible differences between a highly accurate solution based on a more involved simulation, and this approximation. We also used limb darkening data from the terrestrial sun to provide this "appearance feature", even for suns of strongly different temperature. Which is presumably not very realistic, but - as with the unaltered blackbody spectrum probably not a bad first guess, either.

\subsection{Single Stars}

We first conducted experiments for exoplanets illuminated by a single star. For these experiments, we restricted ourselves to Earthlike planets which orbit stars with temperatures between 3000 and 10000 Kelvin. The lower limit of 3000 Kelvin was chosen because the solar disc has, at least if levels of solar radiance comparable to Earth are to be maintained, to get very large beyond this point, which renders the sky dome luminance patterns provided by the Hošek model suspect. As for the upper end of the temperature scale: we stopped at 10000 Kelvin for two reasons. First, due to the hard radiation emitted by much hotter suns, habitable Earth-like planets are an increasingly unlikely proposition as star temperature goes up far beyond this point. And second, as far as visible radiation is concerned, the appearance of outdoor scenes does not seem to
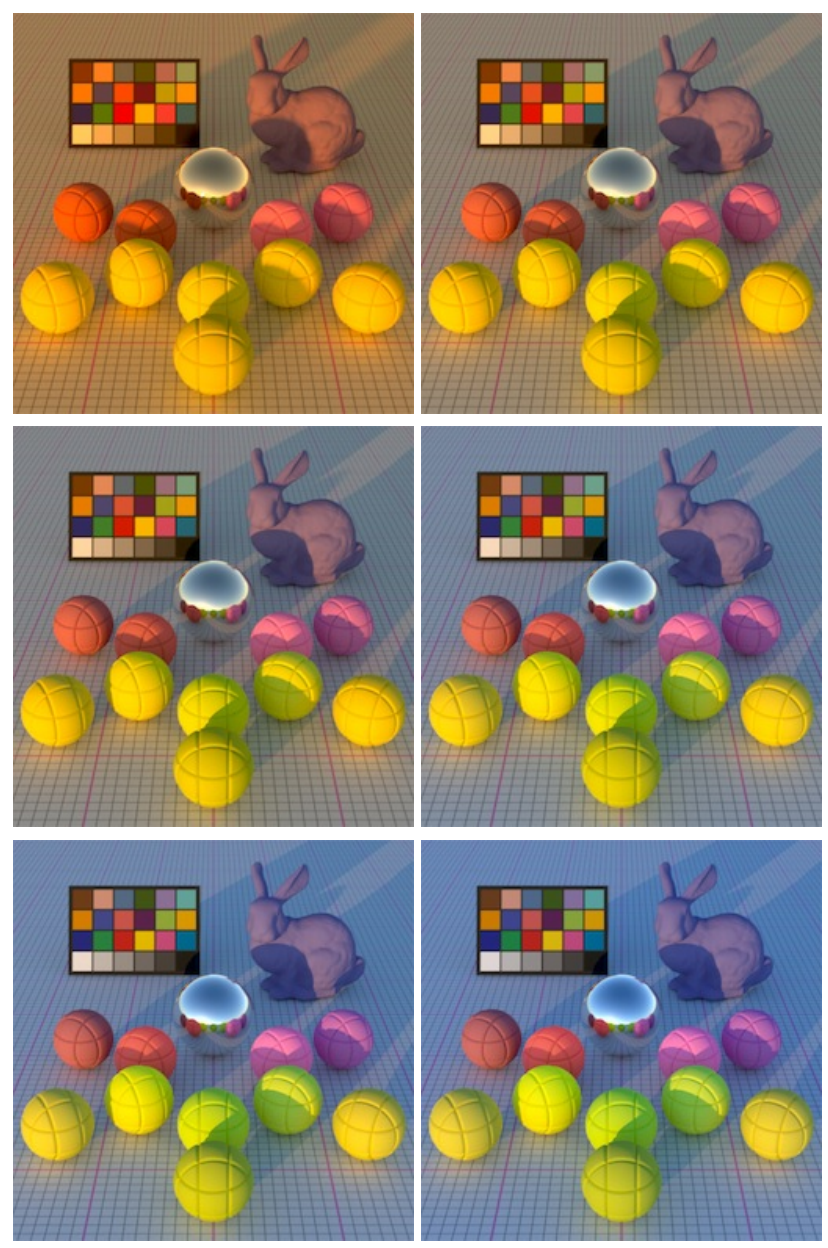

Figure 5: Sunrise/sunset on an exoplanet illuminated by a sun with $3 \mathrm{k}, 4 \mathrm{k}, 5 \mathrm{k}, 6.5 \mathrm{k}, 8 \mathrm{k}$ and $10 \mathrm{k}[\mathrm{K}]$ surface temperature. Note the fact that the sunset still has a definite yellow tinge to it even for a star with 10k temperature, even though the star is blue-white. Solar elevation is 5 degrees, turbidity is 2 . No white balance was applied.

change that much beyond 10000 Kelvin. If similar levels of solar radiation as on Earth are desired, the solar disc shrinks even further towards a point light source for higher temperatures, and the levels of UV radiation rise even further. But the overall colour does not significantly shift towards the blue anymore beyond this point.

Figures 5, 6 and 7 show the obtained results for low and high solar elevations. Two aspects of these results are noteworthy, since they were not expected:

1. As it turns out, sunsets and sunrises on Earth-like exoplanets are still distinctly yellow-orange, even for blue-white stars. The overall appearance to a human observer would of course still be noticeably different, mainly due to the tiny size of the solar disc, and the more intensely blue sky. However, one would still observe a distinct "sunrise/sunset" yellow, not as unlike to scenes seen on Earth as one might assume. This incidentally even holds for brightly blue stars with $40 \mathrm{k} \mathrm{K}$ surface temperature: even such suns would still produce yellow sunsets when viewed through a terrestrial atmosphere.

2. If one performs white balance computations on scenes with a higher solar elevation (which is not an unreasonable thing to do, given the way human vision works), the remaining ap- 

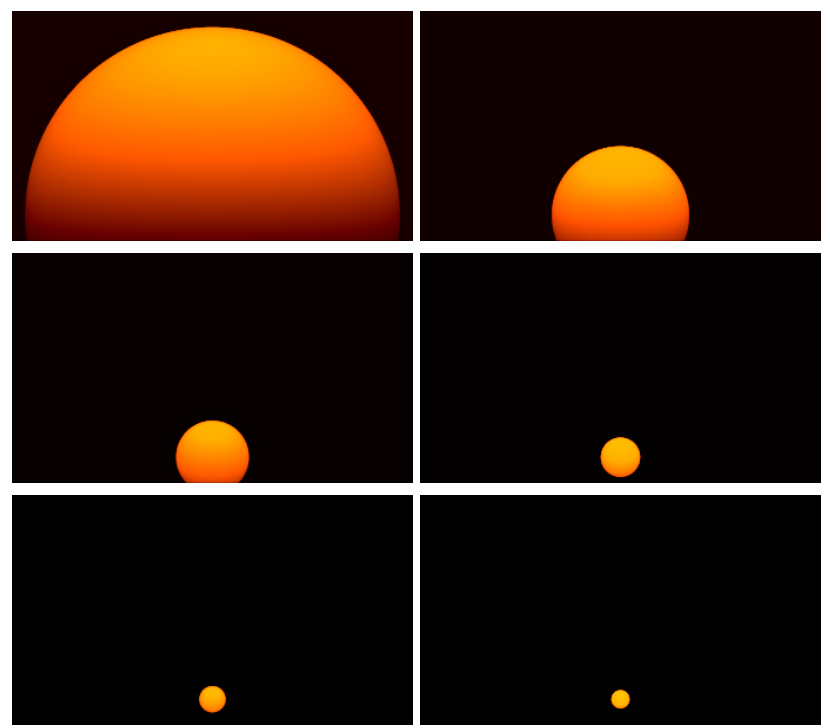

Figure 6: View into the setting sun, elevation $0.26^{\circ}$, i.e. exactly when the terrestrial sun touches the horizon. Temperatures as in figure 5 Solar diameter is $3.79^{\circ}, 1.39^{\circ}, 0.73^{\circ}, 0.40^{\circ}, 0.26^{\circ}$ and $0.18^{\circ}$, respectively. Identical zoom level, but different levels of exposure - the hot suns are much brighter than the cool ones.

pearance differences between scenarios illuminated by suns of varying solar temperature are surprisingly small. Shadows get to be a bit fuzzier for cooler suns (due to the solar discs being larger), while shadows grow very hard for hotter suns (almost like those caused by a point light source). Also, as expected, fluorescent surfaces light up brilliantly under hotter suns, due to the much larger amount of UV radiation present there. However, apart from these differences, the remainder of the appearance is not that dissimilar. In particular, the blue of the sky does grow weaker for cooler suns, but not by as much as one might expect.

The second point has to be taken with a grain of salt insofar as white balance computations only go so far in determining how a human observer immersed in a scene would perceive it. But on balance, the lack of any spectacular changes is not that surprising, if one recalls that when one is speaking of "red" and "blue" suns, one is not talking about very saturated colours to begin with. The blackbody colour sequence that is at the root of star colours is a succession of comparatively weakly saturated colours - otherwise people would not use exactly the same colours as white points (!) for monitors.

One additional issue that is not visible from rendered images, but that would in all likelihood significantly affect the perception of human observers on the ground, is the fact that the tiny solar disc for hotter suns would be even more dangerous to observe with the naked eye than the terrestrial sun: it is smaller in angular diameter, but per our assumptions explained in section 3.1 it would overall emit the same amount of radiation as the terrestrial sun, and even more UV.

\subsection{Binary Stars}

Using the exoplanet solar radiance model described in this paper for scenarios that involve two or more suns is straightforward: light is additive, so one simply uses two models, one for each solar ele- vation and emission spectrum, and adds them together. Care has to be taken to divide the incoming radiation properly: for example, to obtain the same levels of solar radiation as on Earth, each individual sun in a binary sky must only contribute e.g. $50 \%$ of the energy. The exact ratio is of course up to the person doing the modelling, but it should not greatly exceed $100 \%$ of the radiation received on Earth for the reasons outlined in section 3.1 In practice, this will mean that the normal solar disc is replaced by two smaller ones, usually of dissimilar size. The same formula to shrink the solar diameter as discussed in section 3.1 can be used.

To showcase this part of our case study, we chose two hypothetical planets from Science Fiction literature: Tatooine and Solaris.

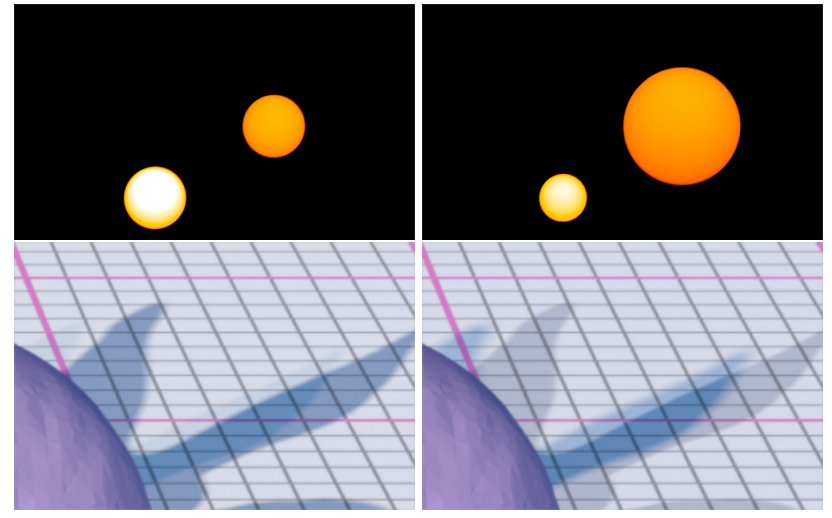

Figure 8: Two cases of dissimilar close binary stars: class $\mathrm{K}$ and $\mathrm{G}$ stars with $4 \mathrm{k}$ and $5.6 \mathrm{k}$ surface temperature. On top, the two suns near sunset. The $\mathrm{G}$ star is over-exposed so that the $\mathrm{K}$ star is still reasonably bright. Below, an enlargement of the shadows cast by them in figures 9 and 10 Left: the case from figure 9 which corresponds to the fictional planet Tatooine in that two suns of different colour and intensity are of equal size on the sky dome. Right: figure 10 the same two suns, scaled so that they are of equal intensity. White balance has been applied to these images.

\subsubsection{Tatooine}

For this experiment, we assumed two suns, of $4 \mathrm{k}$ and $5.6 \mathrm{k}$ surface temperature, i.e. class $\mathrm{K}$ and $\mathrm{G}$ stars. We used them in two configurations, which are shown side by side in figure 8 In the first, both suns are of equal size. Due to the sun with $4 \mathrm{k}$ surface temperature being significantly cooler and less luminous than the $5.6 \mathrm{k}$ one, this leads to the two suns contributing $14 \%$ and $86 \%$ of incident energy, respectively - figure 9 shows the appearance of the test scene under this illumination. In the second configuration, each of them contribute $50 \%$ of the incident energy. The first configuration is what was assumed in the Star Wars movie, as the classical sunset scene shown in figure 2 shows two suns of similar size and different colour. However, we were also interested in what the shadows would look like under such a close binary with two equally bright suns, so we investigated the second configuration as well.

The experiment yielded the interesting insight that both the binary illumination and the differing solar colour are rather inconspicuous in the classical scenario with two similar-sized suns. As can be seen in figure 9 and in close-up also in figure 8 , the shadow fringes are somewhat bi-coloured, but not strongly so. As can be seen in figure 10 however, binary shadows would be a very distinctive feature in all outdoor scenes for the case with two equally bright suns. Also, we suspect (but cannot show this, due to limitations of our renderer) that strong specular reflection highlights, as well as lens 


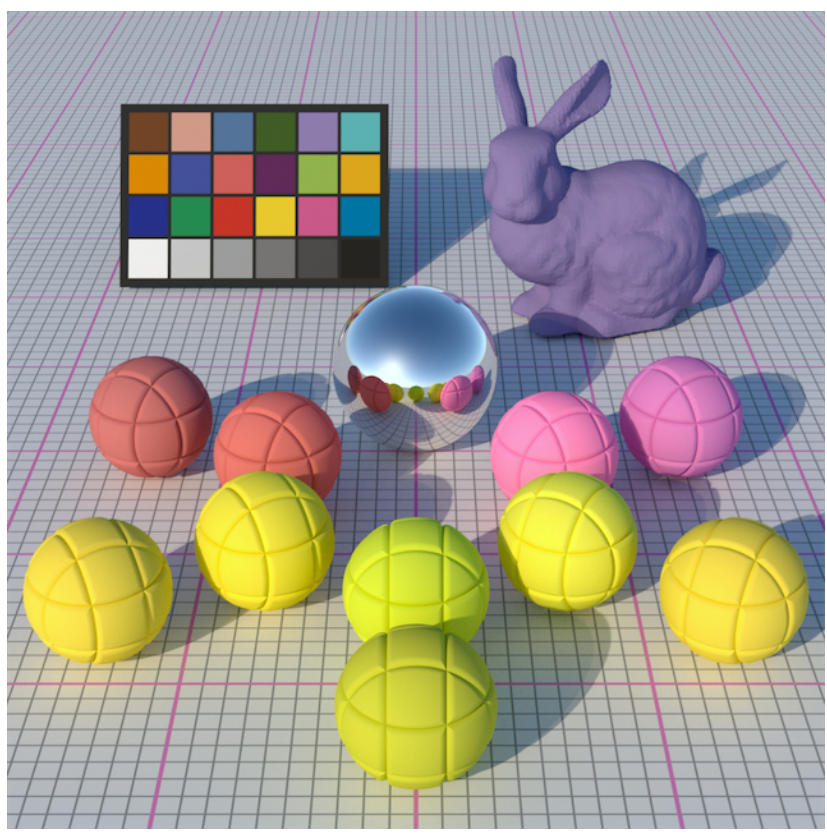

Figure 9: Exoplanet in a binary system, case 1a: two comparatively similar suns (4k and 5.6k) in a close binary constellation, with both stars being of equal size on the sky dome. Turbidity is 3 . The star colours correspond to the data given for the fictional planet Tatooine in Star Wars. When comparing this to the terrestrial shot seen in figure 2, note the off-colour shadow fringes which are noticeably absent from the film footage. Also note the only very subtle colour difference between the two shadows. White balance has been applied to this image.

flares, would be noticeably different under such an illumination: the binary lens flares would probably be very noticeable in both cases.

\subsubsection{Solaris}

Including the planet from Stanisław Lem's seminal novel Solaris in our study is a stretch insofar as the book never mentions that the planet has a breathable atmosphere similar to that of Earth (which is a fundamental assumption in our entire case study). The book features a planet that orbits two strongly dissimilar suns: one red, and one blue. The strange appearance of binary sunrises, and the different colours of the light of the two suns, are explicitly referred to in the story, which made this an attractive experiment regardless. The book also notes that a planet orbiting such a wide and dissimilar binary would not be on a stable orbit - indeed, the fact that the planet is capable of being in any sort of long-term orbit around the given constellation is a mystery in itself that is discussed, but not solved, in the book.

For our experiment, we again chose two suns of equal brightness, with $9.8 \mathrm{k}$ and $2.6 \mathrm{k}$ surface temperature. The appearance of the resulting scene shown in figure 11 is interesting as that after the application of white balance, the appearance is still fairly normal the sky is blue, and the dissimilar colours of the two suns almost cancel out. A notable point are the shadows, which are distinctly bi-coloured in this case - as mentioned in the book. However, one additional point that is not mentioned in the book is also evident: that the shadows show a very noticeable difference in "sharpness", due to the large difference in solar disc size.

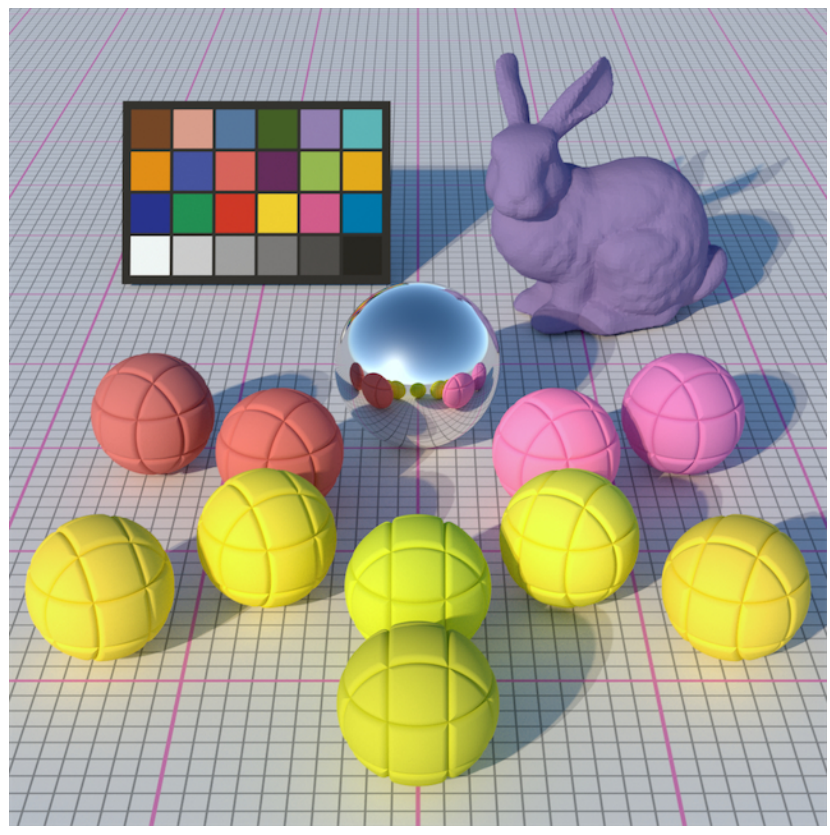

Figure 10: Exoplanet in a binary system, case 1b: two comparatively similar suns ( $4 \mathrm{k}$ and $5.6 \mathrm{k}$ ) in a close binary constellation, with both stars being equally bright. Turbidity is 3 . Note how the doubled shadow edges, as well as the difference in solar colour, are much more noticeable in this scenario than in figure 9 White balance has been applied to this image.

\section{Conclusion}

We have presented a case study on the use of a physically plausible spectral model of sky dome radiance for the prediction of sky dome appearance on one very specific type of exoplanet. Somewhat surprisingly, it demonstrated that for exoplanets of similar size and atmospheric set-up as our home planet, the appearance of outdoor scenes will in all likelihood be very similar to that seen on Earth. Which up to a point stands to reason, as one dominating factor in sky dome appearance is the atmosphere itself, which is explicitly assumed to be identical. But it is nevertheless interesting that the other major input factor, namely different star colours, would appear to play a less significant role in the overall appearance as one might assume, especially if white balance is factored in.

The exoplanet sky dome computations showcased in this paper might conceivably be useful for exobiological computations, e.g. for computations that attempt to determine the habitability of exoplanets for various lifeforms. The re-scaled Hošek model is capable of delivering reasonably plausible estimates for solar irradiance, in particular for UV incidence (which can be a limiting factor for life as we know it).

In the future, we plan to investigate other atmospheric configurations by re-running the brute force path tracer at the core of the Hošek model for explicitly simulated alien atmospheres that are dissimilar to the atmosphere on Earth.

\section{References}

Ashley, M. 2000. The History of the Science Fiction Magazine. Liverpool University Press. 


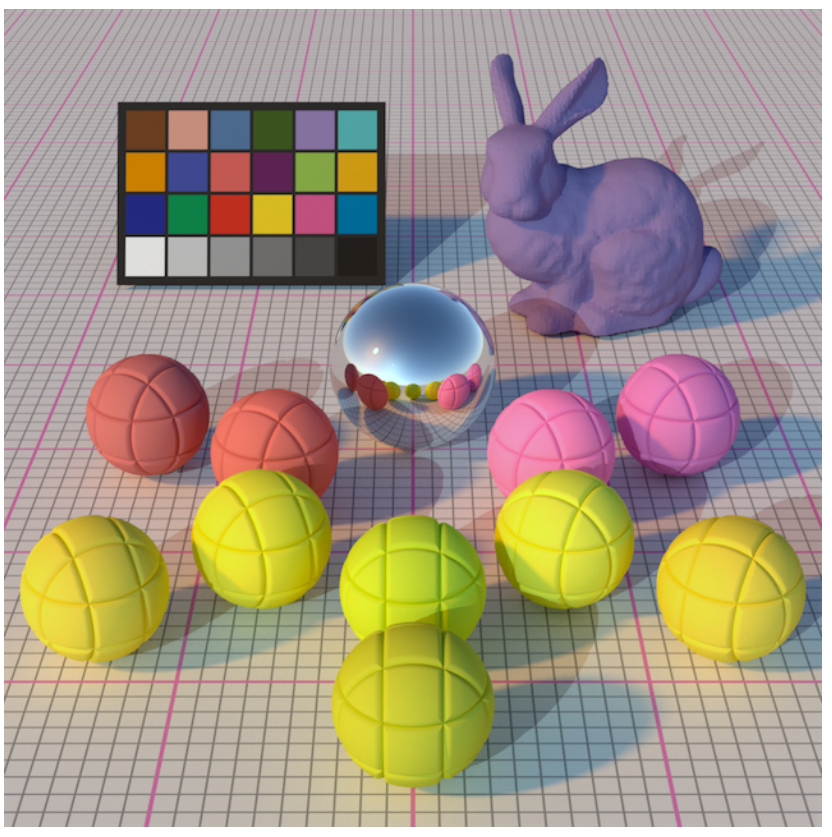

Figure 11: Exoplanet in a binary system, case 2: two dissimilar suns (9.8k and 2.6k) in a distant constellation. Turbiditiy is 3 . This roughly corresponds to the data given for the fictional planet Solaris in the eponymous novel. Note the seemingly paradoxical shadows: the large red sun causes fuzzy blue shadows, while the tiny blue one causes hard-edged red shadows. Also note the fluorescence caused by the blue sun, the still not extremely strong colour difference between the shadows, and the overall still fairly normal appearance of the sky. White balance has been applied to this image.

DenNis, J. 1995. The world according to Pliny the Elder. Smithsonian 26, 8 (Nov.).

FreItAS, R. 1979. Xenology - An Introduction to the Scientific Study of Extraterrestrial Life, Intelligence, and Civilization. Xenology Research Institute, Sacramento, CA.

Haber, J., Magnor, M., And Seidel, H.-P. 2005. Physicallybased simulation of twilight phenomena. ACM Trans. Graph. 24 (October), 1353-1373.

Hosek, L., And Wilkie, A. 2012. An Analytic Model for Full Spectral Sky-dome Radiance. ACM Trans. Graph. 31, 4.

Hosek, L., And Wilkie, A. 2013. Adding a Solar Radiance Function to the Hosek Skylight Model. IEEE Computers Graphics \& Applications special issue on scattering, to appear.

ISO/CIE. 2004. Technical standard ISO 15469:2004 (CIE S 011/E:2003) "Spatial distribution of daylight - CIE standard general sky". Jointly published by ISO and CIE.

Moreland, C., And Bannister, D. 1989. Antique Maps. Phaidon Press.

NASA, 2013. NASA Exoplanet Science Institute. http:// nexsci.caltech.edu

Preetham, A. J., Shirley, P., And Smits, B. 1999. A practical analytic model for daylight. In Proceedings of the 26th annual conference on Computer graphics and interactive techniques, ACM Press/Addison-Wesley Publishing Co., New York, NY, USA, SIGGRAPH '99, 91-100.
Siegel, R., AND Howell, J. R. 2001. Thermal Radiation Heat Transfer, 4th Edition. Taylor \& Francis, New York, NY. 

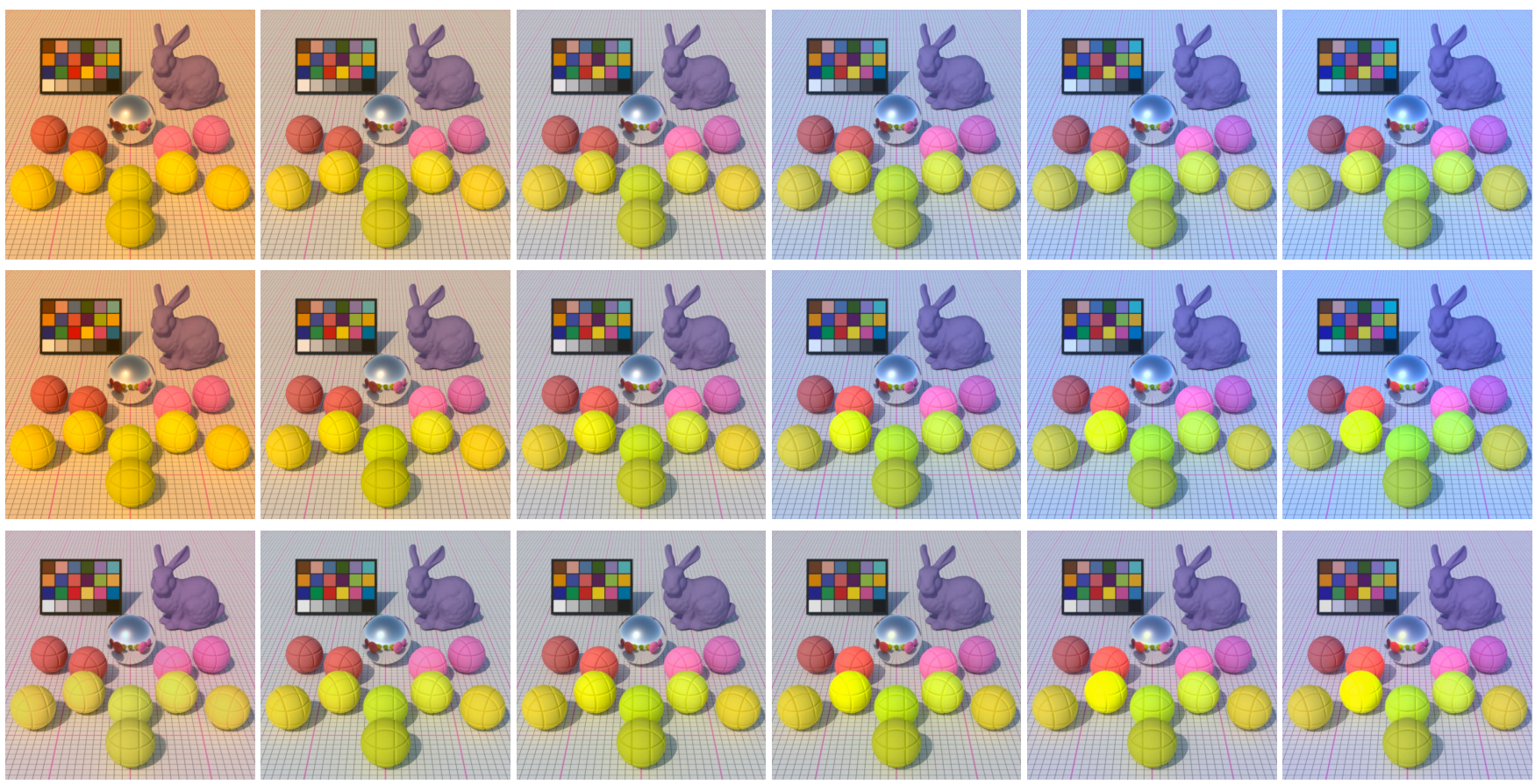

Figure 7: Higher solar elevation $\left(48^{\circ}\right)$ on an exoplanet illuminated by a sun with $3 \mathrm{k}, 4 \mathrm{k}, 5 \mathrm{k}, 6.5 \mathrm{k}, 8 \mathrm{k}$ and $10 \mathrm{k}$ surface temperature, with atmospheric turbidity 5. Top row: computations performed only in the visible range, no white balance. Middle row: computations done including the near UV range, no white balance. Note how compared to visible-only renderings, the fluorescent spheres "light up" for increasing solar temperature, due to the significantly larger amount of UV radiation. Bottom row: the middle row with white balance applied to it, aimed at the white patch of the colour checker. Note how similar the appearance of the scenes becomes once chromatic adaptation is taken into account. The only significant differences that remain are the somewhat softer shadows for lower temperature suns (due to the solar disc being larger), and the presence of fluorescence for hotter suns. Also, the sky is still somewhat (but not dramatically) darker and less blue for cooler suns. 